Original Research Paper

\title{
On Concomitants of Ordered Ranked Set Sampling from Morgenstern Family
}

\author{
${ }^{1,2}$ Mohammed S. Kotb \\ ${ }^{I}$ Department of Mathematics, Faculty of Science, Al-Azhar University, Nasr City, Cairo 11884, Egypt \\ ${ }^{2}$ Department of Mathematics, Faculty of Sciences and Arts-Almikwah, Albaha University, Saudi Arabia
}

\author{
Article history \\ Received: 23-09-2017 \\ Revised: 23-11-2017 \\ Accepted: 27-12-2017
}

Email: msakotb1712@yahoo.com

\begin{abstract}
This paper develops the distribution theory of concomitants in the context of ordered ranked set sample from the Farlie-GumbelMorgenstern bivariate family. Here, we derive the joint distribution of concomitants of two ordered ranked set sample and obtain their single and product moments alongside the correlation coefficient. Finally, an application of the results in establishing the moments and the variancecovariance for the Gumbel's bivariate exponential distribution.
\end{abstract}

Keywords: Concomitants, Gumbel's Bivariate Exponential, Morgenstern Family, Ordered Ranked Set Sampling

\section{Acronyms and Notations}

\begin{tabular}{|c|c|}
\hline FGM & Farlie-Gumbel-Morgenstern. \\
\hline RSS & Ranked Set Sampling. \\
\hline ORSS & Ordered Ranked Set Sample. \\
\hline SRS & Simple Random Sampling. \\
\hline pdf & probability density function. \\
\hline df & distribution function. \\
\hline IID & Independent and \\
\hline & Distributed. \\
\hline INID & Independent and Non-Identically \\
\hline & Distributed. \\
\hline r.v.'s & random variables. \\
\hline$X_{r: n}$ & $\begin{array}{l}r \text {-th ordered failure time when the } \\
\text { sample size is } n \text {. }\end{array}$ \\
\hline$X_{i: n}^{\text {ORSS }}$ & $i$-th ORSS. \\
\hline$\mu_{i: n}^{O R S S}, \sigma_{i: n}^{O R S S}$ & mean and variance of $X_{i: n}^{\text {ORSS }}$ \\
\hline$Y_{[r: n]}$ & $r$-th concomitant of order statistics. \\
\hline$Y_{[r: n]}^{\text {ORSS }}$ & $r$-th concomitant of ORSS. \\
\hline
\end{tabular}

\section{Introduction}

The concept of RSS, was first suggested by McIntyre (1952), has been introduced as a method to increase the precision of estimated yield without the bias of researcherchosen samples and reduce cost by using simple judgment or qualitative information. RSS is a cost-efficient alternative to SRS, particularly in situations where the measurements on the selected subject are expensive or difficult to obtain, see Yu (2001). Many authors have tried to evaluate the performance of RSS, see for example Halls and Dell (1966), Martin et al. (1980) and Alodat and Al-Sagheer (2007). Takahasi and Wakimoto (1968) have presented a more detailed mathematical development for the RSS. During the last three decades, there has been much work on RSS to improve and study the estimation of population parameters, see for example Sinha et al. (1996), Samawi et al. (1996), Adatia (2000), Al-Saleh and Al-Omari (2002), Shaibu and Muttlak (2004), Balakrishnan and Li (2008), Kadilar et al. (2009), Al-Saleh and Samawi (2010), Ozturk (2011), Mohie El-Din et al. (2015) and Sadek et al. (2015). The prediction problems based on RSS are studied by several authors, for instance Salehi et al. (2015), Kotb (2016), Mohie El-Din et al. (2017) and Kotb and Raqab (2017).

Let $X_{1}, X_{2}, \cdots, X_{n}$ be a sequence of IID r.v.'s of size $n$ with a continuous distribution $F$. McIntyre's concept depends on measuring, the smallest observation from the first sample (denoted as $X_{11}$ ), the second smallest observation $X_{22}$ from the second sample and so on until the maximum observation $X_{n n}$ from the last sample. We call this process as a one-cycle RSS of size $n$ and the measured RSS units are denoted by $X_{11}, X_{22}, \cdots, X_{n n}$. By repeating this process independently $m$ times, we obtain secondary sample of size $\mathrm{nm}$ form RSS.

The RSS estimator $\tilde{\mu}_{R S S}$ and variance of the population mean are given by:

$$
\tilde{\mu}_{R S S}=\frac{1}{n} \sum_{i=1}^{n} X_{i i} \text { and } \operatorname{Var}\left(\tilde{\mu}_{R S S}\right)=\frac{\sigma^{2}}{n}-\frac{1}{n^{2}} \sum_{i=1}^{n}\left(\mu_{i i}-\mu\right)^{2} \text {. }
$$


McIntyre (1952) showed that average yield of pasture $\tilde{\mu}_{R S S}$ is unbiased estimator. Takahasi and Wakimoto (1968) studied the variance of $\tilde{\mu}_{R S S}$ and showed that $\operatorname{Var}\left(\tilde{\mu}_{R S S}\right)<\operatorname{Var}\left(\tilde{\mu}_{S R S}\right)$, where $\tilde{\mu}_{S R S}$ and $\tilde{\mu}_{R S S}$ are unbiased estimators for the population mean $\mu$ of a sample of size $n$ obtained from the SRS and the RSS schemes, respectively. They showed also that:

$$
1 \leq e f f\left(\tilde{\mu}_{R S S}, \tilde{\mu}_{S R S}\right)=\frac{\operatorname{Var}\left(\tilde{\mu}_{S R S}\right)}{\operatorname{Var}\left(\tilde{\mu}_{R S S}\right)} \leq \frac{n+1}{2} .
$$

In addition, Stokes (1980) showed that:

$$
1 \leq \frac{\operatorname{Var}\left(\tilde{\sigma}_{S R S}^{2}\right)}{\operatorname{MSE}\left(\tilde{\sigma}_{R S S}^{2}\right)}, \text { for } n \text { sufficiently large, }
$$

where, $\tilde{\sigma}_{S R S}^{2}$ and $\tilde{\sigma}_{R S S}^{2}$ are usual unbiased estimators of $\sigma^{2}$ from the SRS and the RSS schemes of size $n$, respectively.

The idea of RSS based on order statistics from INID r.v.'s has been introduced by Balakrishnan and Li (2008). They showed that the BLUE based on ORSS are more efficient than the BLUE based on RSS for the normal, logistic and two-parameter exponential distributions.

Now, let the $n$ pairs $\left(X_{i}, Y_{i}\right)$ be IID r.v.'s drawn from a bivariate distribution with $\mathrm{df} F(x, y)$. If the $\left(X_{i}, Y_{i}\right)$ are ordered by their $X$-variates (in increasing order of magnitude), then the $Y$ values associated with the $X_{r: n}$, $1 \leq r \leq n$ of $X$ (denoted as $\left.Y_{[r: n]}\right)$ is called the concomitant of the $r$-th order statistics.

The paired $(X, Y)$ is said to be a bivariate FGM distributed if the bivariate df $F(x, y)$ is an absolutely continuous such that:

$$
F_{X, Y}(x, y)=F_{X}(x) F_{Y}(y)\left(1+\alpha\left(1-F_{X}(x)\right)\left(1-F_{Y}(y)\right)\right),
$$

where, $-1 \leq \alpha \leq 1$. The corresponding pdf is given by:

$$
f_{X, Y}(x, y)=f_{X}(x) f_{Y}(y)\left(1+\alpha\left(2 F_{X}(x)-1\right)\left(2 F_{Y}(y)-1\right)\right) \text {. }
$$

Therefore, the conditional df and pdf of $Y$ given $X=x$ are given by:

$$
F_{Y \mid X}(y \mid x)=F_{Y}(y)\left(1+\alpha\left(1-2 F_{X}(x)\right)\left(1-F_{Y}(y)\right)\right),
$$

and:

$$
f_{Y \mid X}(y \mid x)=f_{Y}(y)\left(1+\alpha\left(2 F_{X}(x)-1\right)\left(2 F_{Y}(y)-1\right)\right),
$$

respectively. This family, was first suggested by Morgentern (1956), is characterized by the specified marginal dfs $F_{X}(x)$ and $F_{Y}(y)$ of r.v.'s $X$ and $Y$, respectively. Gumbel (1960) investigated and studied this model for exponential marginals. The FGM is a flexible family useful in applications provided the correlation between the r.v.'s is not too large. References on this family may be found, among many others in Farlie (1960), Johnson and Kott (1975; Johnson and Kotz, 1977), Bairamov and Bekci (1999) and Beg and Ahsanullah (2008). A wide variety of applications for concomitants been used in many fields such as inference problems, ocean engineering, selection procedure, double sampling plans and prediction analysis. These applications for concomitants were introduced by Gross (1973), O'Connel and David (1976), Yang (1977), Yeo and David (1984), Castillo (1988), Do and Hall (1991), Balasubramanian and Beg (1997) and Domma and Giordano (2015). For comprehensive and superb review of work on concomitants of order statistics, we refer the reader to the Handbook of Statistics by David and Nagaraja (1998).

The rest of the present paper is organized as follows: The next Section contains some preliminaries. In section 3, we use the idea of order statistics from INID r.v.'s to derive the pdf, joint pdf and distribution of the concomitants from the FGM bivariate family (1) based on ORSS. In section 4, we obtain the single moments, product moments and the variance-covariances of the concomitants of ORSS. In section 5, the Gumbel's (1960) bivariate exponential distribution are used as special case of our results. We end the paper with a short conclusion in section 6 .

\section{Preliminaries}

Let the pairs $\left(X_{i}, Y_{i}\right), i=1,2, \cdots, n$ be a sequence of IID random variables with df $F(x, y)$. This sequence will be referred to here as a SRS. Let $X_{j 1}, X_{j 2}, \cdots, X_{j n}, j=1$, $2, \cdots, n$ be the visual (judgment)-ordered observations of $n$ sets within a one-cycle. The procedure of a one-cycle RSS can be described in the following Scheme 1:

$$
\begin{aligned}
& X_{11} \leq X_{12} \leq \cdots \leq X_{1 n} \rightarrow X_{11} \\
& X_{21} \leq X_{22} \leq \cdots \leq X_{2 n} \rightarrow X_{22} \\
& X_{n 1} \leq X_{n 2} \leq \cdots \leq X_{n n} \rightarrow X_{n n} \\
& \text { Judgment Rank } \underbrace{}_{\text {RSS }}
\end{aligned}
$$

Scheme 1: A ranked set sample design with sample size $n$

Also, we will need the following definition in the next section to obtain the joint pdf of the concomitants of ORSS.

\section{Definition 1}

Let $B=\left(b_{i, j}\right)$ be a real square matrix of size $n \times n$. The permanent of $B$ is defined to be the scalar:

$$
\operatorname{PerB}=\sum_{P} \prod_{j=1}^{n} b_{j, i_{j}},
$$


where, $\Sigma_{P}$ denotes the summation over all $n$ ! permutations $\left(i_{1}, i_{2}, \cdots, i_{n}\right)$ of $(1,2, \cdots, n)$.

If we arrange the $X^{\prime}$ 's in ascending $X_{\text {ORSS }}=X_{1: n}^{\text {ORSS }} \leq$ $X_{2: n}^{\text {ORSS }} \leq \cdots \leq X_{n: n}^{\text {ORSS }}$ where $X_{i: n}^{\text {ORSS }}$ is the $i$ th ORSS from a continuous population $F(x, y)$, then the $Y$ 's associate with these ORSS are denoted by $Y_{[1]}^{\text {ORSS }}, Y_{[2]}^{\text {ORSS }}, \cdots, Y_{[n]}^{\text {ORSS }}$ and are called concomitants of ORSS. By using the results for order statistics from INID r.v.'s (see Balakrishnan (2008) and Balakrishnan and $\mathrm{Li}(2008)$ ), the one dimensional marginal density function of ORSS is given by:

$$
f_{r: n}^{O R S S}(x)=\sum_{P} \sum_{\underline{i}, \underline{j}}^{n} c_{i, j}(r) f_{\tilde{r}: n^{2}}(x), \quad x \in \Re,
$$

where, $\Sigma_{\mathrm{P}}$ was defined in Definition 1 ,

$$
\begin{aligned}
\sum_{\underline{i}, \underline{j}}^{n} & =\sum_{j_{1}=i_{1}}^{n} \ldots \sum_{j_{r-1}=i_{r-1}}^{n} \sum_{j_{r+1}=0}^{i_{r+1}-1} \cdots \sum_{j_{n}=0}^{i_{n}-1} \\
c_{i, j}(r) & =\frac{1}{(r-1) !(n-r) !}\left(\prod_{k=1, k \neq r}^{n}\left(\begin{array}{l}
n \\
j_{k}
\end{array}\right)\right) i_{r}\left(\begin{array}{l}
n \\
i_{r}
\end{array}\right) \frac{(\tilde{r}-1) !\left(n^{2}-\tilde{r}\right) !}{\left(n^{2}\right) !} \\
\tilde{r} & =i_{r}+\sum_{k=1, k \neq r}^{n} j_{k},
\end{aligned}
$$

and $f_{\tilde{r}: n^{2}}(x)$ is the density of $Y_{\tilde{r}: n^{2}}$, see Arnold et al. (1992) and David and Nagaraja (2003).

The following function is the two dimensional marginal density function of ORSS:

$$
\begin{aligned}
& f_{r, s: n}^{\text {ORSS }}\left(x_{1}, x_{2}\right) \\
& \quad=\sum_{P} \sum_{\underline{i}, \underline{j}, \underline{k} \underline{n}}^{n} c_{i, j, k}(r, s) f_{r^{\prime}, s^{\prime}: n^{2}}\left(x_{1}, x_{2}\right),-\infty<x_{1}<x_{2}<\infty,
\end{aligned}
$$

see Balakrishnan and Li (2008), where:

$$
\begin{aligned}
& \sum_{\underline{i}, \underline{j}, \underline{\underline{k}}}^{n}=\sum_{j_{1}=i_{1}}^{n} \cdots \sum_{j_{s-1} i_{s-1}}^{n} \sum_{j_{s}=0}^{i_{s}-1} \cdots \sum_{j_{n}=0}^{i_{n}-1} \sum_{k_{1}=0}^{n-j_{1}} \cdots \sum_{k_{r-1}=0}^{n-j_{r-1}} \\
& \times \sum_{k_{r+1}=j_{r+1}+1-i_{r+1}}^{j_{r}+1} \ldots \sum_{k_{s-1}=j_{s-1}+1-i_{s-1}}^{j_{s-1}} \sum_{k_{s+1}=0}^{j_{s+1}} \cdots \sum_{k_{n}=0}^{j_{n}}, \\
& c_{i, j, k}(r, s)=\tilde{c}_{i, j, k}(r, s) \frac{\left(r^{\prime}-1\right) !\left(s^{\prime}-r^{\prime}-1\right) !\left(n^{2}-s^{\prime}\right)}{(r-1) !(s-r-1) !(n-r) !\left(n^{2}\right) !}, \\
& \tilde{c}_{i, j, k}(r, s)=\left(\prod_{v=1}^{r-1}\left(\begin{array}{l}
n \\
j_{v}
\end{array}\right)\left(\begin{array}{c}
n-j_{v} \\
k_{v}
\end{array}\right)\right)\left(i_{r}\left(\begin{array}{l}
n \\
i_{r}
\end{array}\right)\left(\begin{array}{c}
n-i_{r} \\
j_{r}-i_{r}
\end{array}\right)\right) \\
& \times\left(\prod_{v=r+1}^{s-1}\left(\begin{array}{l}
n \\
j_{v}
\end{array}\right)\left(\begin{array}{l}
j_{v} \\
k_{v}
\end{array}\right)\right)\left(i_{s}\left(\begin{array}{l}
n \\
i_{s}
\end{array}\right)\left(\begin{array}{c}
i_{s}-1 \\
j_{s}
\end{array}\right)\right)\left(\prod_{v=s+1}^{n}\left(\begin{array}{l}
n \\
j_{v}
\end{array}\right)\left(\begin{array}{l}
j_{v} \\
k_{v}
\end{array}\right)\right), \\
& r^{\prime}=\sum_{v=1, v \neq r, s}^{n} j_{v}+i_{r}+i_{s}-\sum_{v=r+1, v \neq s}^{n} k_{v}-j_{s}-1, \\
& s^{\prime}=\sum_{v=1, y \neq s}^{n} j_{v}+i_{s}+\sum_{v=1}^{r-1} k_{v},
\end{aligned}
$$

and $f_{r^{\prime}, s^{\prime}: n^{2}}\left(x_{1}, x_{2}\right)$ is the joint density function of $X_{r^{\prime}: n^{2}}$ and $X_{s^{\prime} n^{2}}, 1 \leq r^{\prime}<s^{\prime} \leq n^{2}$.

The mean and the variance of $X_{i: n}^{\text {ORSS }}$ are given by:

$$
\mu_{i: n}^{O R S S}=\int_{-\infty}^{\infty} x f_{i: n}^{\text {ORSS }}(x) d x
$$

and

$$
\sigma_{i: n}^{2}=\int_{-\infty}^{\infty}\left(x-\mu_{i: n}^{O R S S}\right)^{2} f_{i: n}^{O R S S}(x) d x .
$$

In addition, the parent pdf and the corresponding mean of RSS can be expressed as:

$$
g(x)=\frac{1}{n} \sum_{i=1}^{n} f_{i: n}^{\text {ORSS }}(x), \quad \mu=\frac{1}{n} \sum_{i=1}^{n} \mu_{i: n}^{\text {ORSS }}
$$

and the corresponding variance is given by:

$$
\sigma^{2}=\frac{1}{n} \sum_{i=1}^{n} \sigma_{i: n}^{2}-\frac{1}{n} \sum_{i=1}^{n}\left(\mu_{i: n}^{\text {ORSS }}-\mu\right)^{2}
$$

\section{Distribution of Concomitants of ORSS}

For the FGM bivariate distributions with df and pdf given by (1) and (2), respectively, the df and pdf of the concomitant of $Y_{[r: n]}^{\text {ORSS }}, 1 \leq r \leq n$ are given, respectively, by:

$$
\left.\begin{array}{r}
F_{Y_{[r: n]}}^{\text {ORSS }}(y)=\int F_{Y \mid X}(y \mid x) f_{r: n}^{\text {ORSS }}(x) d x, \\
f_{Y_{[r: n]}}^{\text {ORSS }}(y)=\int f_{Y \mid X}(y \mid x) f_{r: n}^{\text {ORSS }}(x) d x,
\end{array}\right\}
$$

where, $f_{r: n}^{\text {ORSS }}(x)$ is the density of $X_{r: n}^{\text {ORSS }}$. Utilizing (3), (4) and (6) in (8), we find:

$$
F_{Y_{[r n]]}^{O R S S}}^{\text {of }}(y)=F_{Y}(y)\left(1+\alpha\left(1-F_{Y}(y)\right)\left(1-2 \sum_{P} \sum_{\underline{\underline{i}}, \underline{j}}^{n} c_{i, j}(r) \frac{\tilde{r}}{n^{2}+1}\right)\right) \text {, }
$$

and:

$f_{Y_{[r n]]}}^{\text {ORSS }}(y)=f_{Y}(y)\left(1+\alpha\left(1-2 F_{Y}(y)\right)\left(1-2 \sum_{P} \sum_{\underline{i}, \underline{j}}^{n} c_{i, j}(r) \frac{\tilde{r}}{n^{2}+1}\right)\right)$.

Now, we derive the joint distribution of $r$ th and $s$ th concomitants. The joint df of $\left(Y_{[r: n]}^{\text {ORSS }}, Y_{[s: n]}^{\text {ORSS }}\right), 1 \leq r<s \leq n$ is given by:

$$
\begin{aligned}
& F_{[r, s: n]}^{\text {ORSS }}\left(y_{1}, y_{2}\right) \\
& =\int_{-\infty}^{\infty} \int_{-\infty}^{x_{2}} F_{Y \mid X}\left(y_{1} \mid x_{1}\right) F_{Y \mid X}\left(y_{2} \mid x_{2}\right) f_{[r, s: n]}^{\text {ORSS }}\left(x_{1}, x_{2}\right) d x_{1} d x_{2},
\end{aligned}
$$

where, $f_{r, s: n}^{\text {ORS }}\left(x_{1}, x_{2}\right)$ is given in (7). 
Upon using (3) and (7) in (11) and after some simplification, we obtain:

$$
\begin{aligned}
& F_{[r, s, n]}^{\text {ORS }}\left(y_{1}, y_{2}\right) \\
& =F_{Y}\left(y_{1}\right) F_{Y}\left(y_{2}\right)\left[1+\alpha\left(1-F_{Y}\left(y_{1}\right)\right)\left(1-2 \sum_{P} \sum_{i, j, j, \underline{k}}^{n} c_{i, j, k}(r, s) \frac{r^{\prime}}{n^{2}+1}\right)\right. \\
& -\alpha\left(1-F_{Y}\left(y_{2}\right)\right)\left(1-2 \sum_{P} \sum_{i, j, j, \underline{k}}^{n} c_{i, j, k}(r, s) \frac{n^{2}-s^{\prime}+1}{n^{2}+1}\right) \\
& -\alpha^{2}\left(1-F_{Y}\left(y_{1}\right)\right)\left(1-F_{Y}\left(y_{2}\right)\right) \\
& \left.\times\left(1-2 \sum_{P} \sum_{i, j, j, k}^{n} c_{i, j, k}(r, s)\left\{\frac{n^{2}-s^{\prime}+1}{n^{2}+1}-\frac{2 r^{\prime}\left(n^{2}-2 s\right)}{\left(n^{2}+1\right)\left(n^{2}+2\right)}\right\}\right)\right],
\end{aligned}
$$

and the pdf corresponding to (12) is:

$$
\begin{aligned}
& f_{[r, s, n]}^{\text {ORSS }}\left(y_{1}, y_{2}\right) \\
& =f_{Y}\left(y_{1}\right) f_{Y}\left(y_{2}\right)\left[1+\alpha\left(1-2 F_{Y}\left(y_{1}\right)\right)\left(1-2 \sum_{P} \sum_{i, j, \underline{,}, \underline{k}}^{n} c_{i, j, k}(r, s) \frac{r^{\prime}}{n^{2}+1}\right)\right. \\
& -\alpha\left(1-2 F_{Y}\left(y_{2}\right)\right)\left(1-2 \sum_{P} \sum_{i, j, j, k}^{n} c_{i, j, k}(r, s) \frac{n^{2}-s^{\prime}+1}{n^{2}+1}\right) \\
& -\alpha^{2}\left(1-2 F_{Y}\left(y_{1}\right)\right)\left(1-2 F_{Y}\left(y_{2}\right)\right) \\
& \left.\times\left(1-2 \sum_{P} \sum_{i, j, j, \underline{k}}^{n} c_{i, j, k}(r, s)\left\{\frac{n^{2}-s^{\prime}+1}{n^{2}+1}-\frac{2 r^{\prime}\left(n^{2}-2 s\right)}{\left(n^{2}+1\right)\left(n^{2}+2\right)}\right\}\right)\right] .
\end{aligned}
$$

More generally, the joint density function of $Y_{\left[r_{1}: n\right]}^{\text {ORS }}$, $Y_{\left[r_{2}: n\right]}^{\text {ORS }}, \cdots, Y_{\left[r_{k}: n\right]}^{\text {ORSS }},\left(1 \leq r_{1}<r_{2}<\cdots<r_{k} \leq n\right)$, can be written as:

$f_{\left[r_{1}, \cdots, r_{k}\right]}^{\text {ORSS }}(y)$

$=\int_{-\infty}^{\infty} \int_{-\infty}^{x_{k}} \cdots \int_{-\infty}^{x_{2}} \prod_{i=1}^{k} f_{Y \mid X}\left(y_{i} \mid x_{i}\right) f_{r_{1}, \cdots, r_{k}}^{O R S S}\left(x_{1}, \cdots, x_{k}\right) d x_{1} \cdots d x_{k}$,

where, $\mathrm{y}=\left(y_{1}, \cdots, y_{k}\right)$ and $f_{r_{1}, \cdots, r_{k}}^{\text {ORSS }}\left(x_{1}, \cdots, x_{k}\right)$ is the joint pdf of $\left(X_{r_{1}: n}^{\text {ORSS }}, \cdots, X_{r_{k}: n}^{\text {ORSS }}\right)$.

From Definition 1 and (13), we readily see that the joint pdf can be written as:

$$
\begin{aligned}
& f_{\left[r_{1}, \cdots, r_{k}\right]}^{\text {ORS }}(y)=\frac{1}{d_{k, n}}\left(\prod_{i=1}^{k} f\left(y_{i}\right)\right) \int_{-\infty}^{\infty} \int_{-\infty}^{x_{k}} \cdots \int_{-\infty}^{x_{2}} \operatorname{PerB}_{k}(x) \\
& \times\left(\prod_{i=1}^{k}\left(1+\alpha\left(2 F\left(x_{i}\right)-1\right)\left(2 F\left(y_{i}\right)-1\right)\right)\right) d x_{1} \cdots d x_{k}
\end{aligned}
$$

see Balakrishnan (2008, pp. 19), where $\mathrm{x}=\left(x_{1}, \cdots\right.$, $\left.x_{k}\right), d_{k, n}=\left(r_{1}-1\right) !\left(r_{2}-r_{1}-1\right) ! \cdots\left(r_{k}-r_{k-1}-1\right) !\left(n-r_{k}\right) !$ and
$\operatorname{Per} B_{k}(x)$ is the permanent of the following square Matrix:

$$
\begin{aligned}
& B_{k}(x) \\
& =\left(\begin{array}{ccc}
F_{1}\left(x_{1}\right) & \cdots & F_{n}\left(x_{1}\right) \\
f_{1}\left(x_{1}\right) & \cdots & f_{n}\left(x_{1}\right) \\
F_{1}\left(x_{2}\right)-F_{1}\left(x_{1}\right) & \cdots & F_{n}\left(x_{2}\right)-F_{n}\left(x_{1}\right) \\
f_{1}\left(x_{2}\right) & \cdots & f_{n}\left(x_{2}\right) \\
\vdots & \ddots & \vdots \\
F_{1}\left(x_{k}\right)-F_{1}\left(x_{k-1}\right) & \cdots & F_{n}\left(x_{k}\right)-F_{n}\left(x_{k-1}\right) \\
f_{1}\left(x_{k}\right) & \cdots & f_{n}\left(x_{k}\right) \\
1-F_{1}\left(x_{k}\right) & \cdots & 1-F_{n}\left(x_{k}\right)
\end{array}\right) \begin{array}{l}
\xi r_{1}-1 \\
\xi r_{2}-r_{1}-1 \\
\xi 1 \\
\xi r_{k}-r_{k-1}-1 \\
\xi 1 \\
\xi n-r_{k}
\end{array}
\end{aligned}
$$

\section{Moments of Concomitants Based on ORSS}

Using the results of the previous section, we obtain the single and product moments as well as the variance-covariances of the concomitants of ORSS as follows.

From Equation (10), the $k$ th moment of $Y_{[r: n]}^{\text {ORSS }}, 1 \leq r \leq$ $n$, is given by:

$$
\begin{aligned}
\text { ORSS } \mu_{[r: n]}^{(k)} & =E\left(Y_{[r: n]}^{k}\right)=\int_{-\infty}^{\infty} y^{k} f_{Y_{[r n]}}^{\text {ORSS }}(y) d y \\
& =\mu^{(k)}+\alpha\left(1-2 \sum_{P} \sum_{i, j, \underline{j}}^{n} c_{i, j}(r) \frac{\tilde{r}}{n^{2}+1}\right)\left(\mu^{(k)}-\mu_{2: 2}^{(k)}\right),
\end{aligned}
$$

where, $\mu^{(k)}=E\left(Y^{k}\right), \mu_{2: 2}^{(k)} \equiv E\left(Y_{2: 2}^{k}\right)$ and $k=1,2, \cdots$.

The product moment of two concomitants $\left(Y_{[r: n]}^{\text {ORSS }}, Y_{[s: n]}^{\text {ORSS }}\right), 1 \leq r<s \leq n$, is given by:

$$
\begin{aligned}
& { }_{\text {ORSS }} \mu_{[r, s: n]}^{(k, \ell)}=\mu^{(k)} \mu^{(\ell)} \\
& +\alpha\left(1-2 \sum_{P} \sum_{i, j, \underline{j}, \underline{k}}^{n} c_{i, j, k}(r, s) \frac{r^{\prime}}{n^{2}+1}\right) \mu^{(\ell)}\left(\mu^{(k)}-\mu_{2: 2}^{(k)}\right) \\
& -\alpha\left(1-2 \sum_{P} \sum_{i, j, \underline{j}, \underline{k}}^{n} c_{i, j, k}(r, s) \frac{n^{2}-s^{\prime}+1}{n^{2}+1}\right) \mu^{(k)}\left(\mu^{(\ell)}-\mu_{2: 2}^{(\ell)}\right) \\
& -\alpha^{2}\left(1-2 \sum_{P} \sum_{i, j, j, k}^{n} c_{i, j, k}(r, s)\left\{\begin{array}{l}
\frac{n^{2}-s^{\prime}+1}{n^{2}+1} \\
-\frac{2 r^{\prime}\left(n^{2}-2 s\right)}{\left(n^{2}+1\right)\left(n^{2}+2\right)}
\end{array}\right\}\right) \\
& \times\left(\mu^{(k)} \mu^{(\ell)}-\mu^{(\ell)} \mu_{2: 2}^{(k)}-\mu^{(k)} \mu_{2: 2}^{(\ell)}+\mu_{2: 2}^{(k)} \mu_{2: 2}^{(\ell)}\right) \text {. }
\end{aligned}
$$

Furthermore, using (16) and (17), the variancecovariances of the concomitants of ORSS for $r<s$ is: 


$$
\begin{aligned}
& \sigma_{[r, s: n]}^{\text {ORS }}=\mu^{2}+2 \alpha \mu\left(\mu-\mu_{2: 2}\right) \sum_{P} \sum_{i, j, \underline{j}, \underline{n}}^{n} c_{i, j, k}(r, s) \frac{n^{2}-s^{\prime}-r^{\prime}+1}{n^{2}+1} \\
& -\alpha^{2}\left(\mu-\mu_{2: 2}\right)^{2}\left(1-2 \sum_{P} \sum_{i, j, \underline{j}}^{n} c_{i, j, k}(r, s)\left\{\begin{array}{l}
\frac{n^{2}-s^{\prime}+1}{n^{2}+1} \\
\frac{2 r^{\prime}\left(n^{2}-2 s\right)}{\left(n^{2}+1\right)\left(n^{2}+2\right)}
\end{array}\right\}\right) \\
& -2 \alpha \mu\left(\mu-\mu_{2: 2)}\right)\left(1-\sum_{P} \sum_{i, j, \underline{j}}^{r, n} c_{i, j}(r) \frac{\tilde{r}}{n^{2}+1}-\sum_{P} \sum_{i, j}^{s, n} c_{i, j}(s) \frac{\tilde{s}}{n^{2}+1}\right) \\
& -\alpha^{2}\left(\mu-\mu_{2: 2}\right)^{2}\left(1-\sum_{P} \sum_{i, j}^{r, \underline{n}} c_{i, j}(r) \frac{\tilde{r}}{n^{2}+1}\right)\left(1-\sum_{P} \sum_{i, j, \underline{j}}^{s, n} c_{i, j}(s) \frac{\tilde{s}}{n^{2}+1}\right),
\end{aligned}
$$

and:

$$
\begin{aligned}
& \sigma_{[r, r: n]}^{\text {ORSS }} \\
& =\alpha\left(1-2 \sum_{P} \sum_{i, j}^{n} c_{i, j}(r) \frac{\tilde{r}}{n^{2}+1}\right)\left\{\mu^{(2)}-\mu_{2: 2}^{(2)}-2 \mu\left(\mu-\mu_{2: 2}\right)\right. \\
& \left.+\alpha\left(1-2 \sum_{P} \sum_{i, j}^{n} c_{i, j}(r) \frac{\tilde{r}}{n^{2}+1}\right)\left(\mu-\mu_{2: 2}\right)^{2}\right\} .
\end{aligned}
$$

From Equation (6)-(8) and (13), we can easily show the following:

$$
\begin{aligned}
& E\left(Y_{[r: n]}^{\text {ORSS }}\right)=\sum_{P} \sum_{i, j}^{n} c_{i, j}(r) E\left(E\left[Y_{1} \mid X_{1}=X_{\tilde{r}: n^{2}}\right]\right), \\
& \left.\operatorname{Var}\left(Y_{[r: n]}^{\text {ORSS }}\right)=\sum_{P} \sum_{i, j}^{n} c_{i, j}(r)\left\{\begin{array}{l}
E\left(\operatorname{Var}\left[Y_{1} \mid X_{1}=X_{\tilde{r}: n^{2}}\right]\right) \\
+\operatorname{Var}\left(E\left[Y_{1} \mid X_{1}=X_{\tilde{r}: n^{2}}\right]\right.
\end{array}\right)\right\},
\end{aligned}
$$

and for $r \neq s\left(r^{\prime} \neq s^{\prime}\right)$ :

$$
\begin{aligned}
& \operatorname{Cov}\left(Y_{[r: n]}^{\text {ORSS }}, Y_{[s: n]}^{\text {ORSS }}\right) \\
& =\sum_{P} \sum_{i, j, j, k}^{n} c_{i, j, k}(r, s) \operatorname{Cov}\left(E\left[Y_{1} \mid X_{1}=X_{r^{\prime}: n^{2}}\right], E\left[Y_{1} \mid X_{1}=X_{s^{\prime}: n^{2}}\right]\right) .
\end{aligned}
$$

By using the facts that $\sum_{i=1}^{n} Y_{[i n]]}^{\text {ORSS }}=\sum_{i=1}^{n} Y_{i=1}^{\text {ORSS }}=\sum_{i=1}^{n} Y_{i(i n n)}$, we obtain the following relations immediately:

$$
\begin{aligned}
& \sum_{i=1}^{n} \text { ORSS } \mu_{[i: n]}^{(k)}=\sum_{i=1}^{n} \text { oRSS } \mu_{i: n}^{(k)}=\sum_{i=1}^{n} \mu_{i: n}^{(k)}=n \mu_{1: 1}^{(k)}, \\
& \sum_{i=1}^{n-1} \sum_{j>i} \text { ORSS } \mu_{[i, j: n]}^{(k)}=\sum_{i=1}^{n-1} \sum_{j>i} \text { oRSS } \mu_{i, j: n}^{(k)}=\sum_{i=1}^{n-1} \sum_{j>i} \mu_{i: n}^{(k)} \mu_{j: n}^{(k)},
\end{aligned}
$$

and:

$$
\sum_{i=1}^{n} \text { ORSS } \mu_{[i, i: n]}^{(k)}=E\left(\sum_{i=1}^{n} Y_{i(i: n)}^{2(k)}\right)=n \mu_{1,1: 1 !}^{(k)}
$$

\section{Application}

We consider an RSS $\left(X_{i}, Y_{i}\right), i=1,2, \cdots, n$ from the Gumbel's bivariate exponential distribution of the Morgenstern family (see Gumbel (1960)), whose cdf is given by:

$$
\begin{aligned}
& F_{X, Y}(x, y) \\
& =\left(1-\exp \left(\frac{-x}{\beta_{1}}\right)\right)\left(1-\exp \left(\frac{-y}{\beta_{2}}\right)\right)\left(1+\alpha \exp \left(\frac{-x}{\beta_{1}}+\frac{-y}{\beta_{2}}\right)\right),
\end{aligned}
$$

where, $x, y>0$ and $\beta_{1}, \beta_{2}>0$. Hence, from (16), the moments of $Y_{[r: n]}^{\text {ORSS }}$ is given by:

$$
\begin{aligned}
& \operatorname{ORSS}_{[r: n]}^{(k)} \\
& =\Gamma(k+1)\left\{1+\alpha\left(1-2 \sum_{P} \sum_{\underline{i}, \underline{j}}^{n} c_{i, j}(r) \frac{\tilde{r}}{n^{2}+1}\right)\left(2^{-k}-1\right)\right\} \beta_{2}^{k},
\end{aligned}
$$

and, from (17), the product moment of $Y_{[r: n]}^{\text {ORS }}$ and $Y_{[s: n]}^{\text {ORSS }}$, is given by:

$$
\begin{aligned}
& \text { ORSS } \mu_{[r, s, n]}^{(k, \ell)} \\
& =\Gamma(k+1) \Gamma(\ell+1)\left\{1+\alpha\left(1-2 \sum_{P} \sum_{i, j, j, k}^{n} c_{i, j, k}(r, s) \frac{r^{\prime}}{n^{2}+1}\right)\left(2^{-k}-1\right)\right. \\
& -\alpha\left(1-2 \sum_{P} \sum_{i, j, j, \underline{k}}^{n} c_{i, j, k}(r, s) \frac{n^{2}-s^{\prime}+1}{n^{2}+1}\right)\left(2^{-\ell}-1\right) \\
& -\alpha^{2}\left(1-2 \sum_{P} \sum_{i, j, j, k}^{n} c_{i, j, k}(r, s)\left\{\frac{n^{2}-s^{\prime}+1}{n^{2}+1}-\frac{2 r^{\prime}\left(n^{2}-2 s\right)}{\left(n^{2}+1\right)\left(n^{2}+2\right)}\right\}\right) \\
& \left.\times\left(1+2^{-k-\ell}-2^{-k}-2^{-\ell}\right)\right\} \beta_{2}^{k+\ell} .
\end{aligned}
$$

Consequently the expected value and the variance of $Y_{[r: n]}^{\text {ORSS }}$ can be obtained from Equation (21) as follows:

$$
E\left(Y_{[r: n]}^{\text {ORSS }}\right)=\beta_{2}\left(1-\frac{\alpha}{2}\left(1-2 \sum_{P} \sum_{i, j}^{r, n} c_{i, j}(r) \frac{\tilde{r}}{n^{2}+1}\right)\right),
$$

and:

$$
\begin{aligned}
\operatorname{Var}\left(Y_{[r: n]}^{\text {ORSS }}\right) & =\frac{-\beta_{2}^{2}}{4}\left(\alpha\left(1-2 \sum_{P} \sum_{i, \underline{j}}^{r, n} c_{i, j}(r) \frac{\tilde{r}}{n^{2}+1}\right)+1-\sqrt{5}\right) \\
& \times\left(\alpha\left(1-2 \sum_{P} \sum_{\underline{i}, \underline{j}, \underline{r}}^{r, n} c_{i, j}(r) \frac{\tilde{r}}{n^{2}+1}\right)+1+\sqrt{5}\right) .
\end{aligned}
$$

Furthermore, using (18) and (19), the variancecovariances of $\left(Y_{[r: n]}^{\text {ORSS }}, Y_{[s: n]}^{\text {ORSS }}\right)$ is given by: 


$$
\begin{aligned}
& \sigma_{[r, s: n]}^{\text {ORSS }}=\beta_{2}^{2}\left\{1-\alpha \sum_{P} \sum_{\underline{i}, \underline{j}, \underline{\underline{k}} c_{i, j, k}}^{n}(r, s) \frac{n^{2}-s^{\prime}-r^{\prime}+1}{n^{2}+1}\right. \\
& -\frac{\alpha^{2}}{4}\left(1-2 \sum_{P} \sum_{\underline{i}, \underline{j}, \underline{\underline{k}}}^{n} c_{i, j, k}(r, s)\left\{\frac{n^{2}-s^{\prime}+1}{n^{2}+1}-\frac{2 r^{\prime}\left(n^{2}-2 s\right)}{\left(n^{2}+1\right)\left(n^{2}+2\right)}\right\}\right) \\
& +\alpha\left(1-\sum_{P} \sum_{\underline{i}, \underline{j}}^{r, n} c_{i, j}(r) \frac{\tilde{r}}{n^{2}+1}-\sum_{P} \sum_{\underline{i}, \underline{j}}^{s, n} c_{i, j}(s) \frac{\tilde{s}}{n^{2}+1}\right) \\
& \left.-\frac{\alpha^{2}}{4}\left(1-\sum_{P} \sum_{\underline{i}, \underline{j}}^{r, n} c_{i, j}(r) \frac{\tilde{r}}{n^{2}+1}\right)\left(1-\sum_{P} \sum_{\underline{i}, \underline{j}}^{s, n} c_{i, j}(s) \frac{\tilde{s}}{n^{2}+1}\right)\right\},
\end{aligned}
$$

and:

$$
\begin{aligned}
& \sigma_{[r, r, n]}^{\text {ORSS }} \\
& =\frac{\alpha \beta_{2}^{2}}{2}\left(1-2 \sum_{P} \sum_{\underline{i}, \underline{j}}^{n} c_{i, j}(r) \frac{\tilde{r}}{n^{2}+1}\right)\left\{-1+\frac{\alpha}{2}\left(1-2 \sum_{P} \sum_{\underline{i}, \underline{j}}^{n} c_{i, j}(r) \frac{\tilde{r}}{n^{2}+1}\right)\right\} .
\end{aligned}
$$

By using Equation (23), (25) and (26), the

\begin{tabular}{|c|c|c|c|c|c|c|c|c|c|c|c|}
\hline$n$ & $r$ & $\mu_{r: n}^{\text {ORSS }}$ & $n$ & $r$ & $\mu_{r: n}^{\text {ORSS }}$ & $n$ & $r$ & $\mu_{r: n}^{\text {ORSS }}$ & $n$ & $r$ & $\mu_{r: n}^{\text {ORSS }}$ \\
\hline 1 & 1 & 1.00000 & 4 & 4 & 1.16909 & 6 & 4 & 1.03998 & 7 & 7 & 1.20245 \\
\hline \multirow[t]{2}{*}{2} & 1 & 0.90000 & 5 & 1 & 0.81560 & & 5 & 1.11927 & 8 & 1 & 0.79178 \\
\hline & 2 & 1.10000 & & 2 & 0.90570 & & 6 & 1.19487 & & 2 & 0.84890 \\
\hline \multirow[t]{3}{*}{3} & 1 & 0.85536 & & 3 & 1.00000 & 7 & 1 & 0.79754 & & 3 & 0.90886 \\
\hline & 2 & 1.00000 & & 4 & 1.09430 & & 2 & 0.86262 & & 4 & 0.96956 \\
\hline & 3 & 1.14464 & & 5 & 1.18440 & & 3 & 0.93092 & & 5 & 1.03045 \\
\hline \multirow[t]{3}{*}{4} & 1 & 0.83091 & 6 & 1 & 0.80513 & & 4 & 1.00000 & & 6 & 1.09113 \\
\hline & 2 & 0.94220 & & 2 & 0.88073 & & 5 & 1.06907 & & 7 & 1.15109 \\
\hline & 3 & 1.05780 & & 3 & 0.96002 & & 6 & 1.13737 & & 8 & 1.20821 \\
\hline
\end{tabular}
moments of concomitants of ORSS can be derived from the moments of ORSS for the Gumbel's

\begin{tabular}{|c|c|c|c|c|c|c|c|c|c|c|c|c|c|c|c|}
\hline$n$ & $s$ & $r$ & $\sigma_{r, s: n}^{\text {ORSS }}$ & $n$ & $s$ & $r$ & $\sigma_{r, s: n}^{\text {ORSS }}$ & $n$ & $s$ & $r$ & $\sigma_{r: n}^{\text {ORSS }}$ & $n$ & $s$ & $r a$ & $\sigma_{r: n}^{\text {ORSS }}$ \\
\hline 1 & 1 & 1 & 1.00000 & 5 & 5 & 1 & 0.00002 & 7 & 3 & 2 & 0.00127 & 8 & 4 & 1 & 0.00009 \\
\hline \multirow[t]{3}{*}{2} & 1 & 1 & 0.89000 & & & 2 & 0.00012 & & & 3 & 0.92617 & & & 2 & 0.00046 \\
\hline & 2 & 1 & 0.00306 & & & 3 & 0.00045 & & 4 & 1 & 0.00010 & & & 3 & 0.00128 \\
\hline & & 2 & 1.09000 & & & 4 & 0.00122 & & & 2 & 0.00051 & & & 4 & 0.96863 \\
\hline \multirow[t]{6}{*}{3} & 1 & 1 & 0.83444 & & & 5 & 1.15040 & & & 3 & 0.00153 & & 5 & 1 & 0.00023 \\
\hline & 2 & 1 & 0.00240 & 6 & 1 & 1 & 0.76716 & & & 4 & 1.00000 & & & 2 & 0.00019 \\
\hline & & 2 & 1.00000 & & 2 & 1 & 0.00092 & & 5 & 1 & 0.00001 & & & 3 & 0.00053 \\
\hline & 3 & 1 & 0.00049 & & & 2 & 0.86650 & & & 2 & 0.00002 & & & 4 & 0.00129 \\
\hline & & 2 & 0.00240 & & 3 & 1 & 0.00037 & & & 3 & 0.00058 & & & 5 & 1.02949 \\
\hline & & 3 & 1.12372 & & & 2 & 0.00156 & & & 4 & 0.00157 & & 6 & 1 & 0.00001 \\
\hline \multirow[t]{10}{*}{4} & 1 & 1 & 0.80231 & & & 3 & 0.95842 & & & 5 & 1.06431 & & & 2 & 0.00006 \\
\hline & 2 & 1 & 0.00169 & & 4 & 1 & 0.00011 & & 6 & 1 & 0.00031 & & & 3 & 0.00024 \\
\hline & & 2 & 0.93886 & & & 2 & 0.00062 & & & 2 & 0.00002 & & & 4 & 0.00056 \\
\hline & 3 & 1 & 0.00053 & & & 3 & 0.00177 & & & 3 & 0.00060 & & & 5 & 0.00122 \\
\hline & & 2 & 0.00240 & & & 4 & 1.03838 & & & 4 & 0.00055 & & & 6 & 1.08284 \\
\hline & & 3 & 1.05446 & & 5 & 1 & 0.00003 & & & 5 & 0.00128 & & 7 & 1 & 0.00001 \\
\hline & 4 & 1 & 0.00008 & & & 2 & 0.00017 & & & 6 & 1.11852 & & & 2 & 0.00005 \\
\hline & & 2 & 0.00053 & & & 3 & 0.00062 & & 7 & 1 & 0.00001 & & & 3 & 0.00007 \\
\hline & & 3 & 0.00169 & & & 4 & 0.00156 & & & 2 & 0.00003 & & & 4 & 0.00015 \\
\hline & & 4 & 1.14050 & & & 5 & 1.10505 & & & 3 & 0.00087 & & & 5 & 0.00043 \\
\hline \multirow[t]{10}{*}{5} & 1 & 1 & 0.78159 & & 6 & 1 & 0.00001 & & & 4 & 0.00010 & & & 6 & 0.00096 \\
\hline & 2 & 1 & 0.00122 & & & 2 & 0.00002 & & & 5 & 0.00032 & & & 7 & 1.12827 \\
\hline & & 2 & 0.89680 & & & 3 & 0.00012 & & & 6 & 0.00071 & & 8 & 1 & 0.00001 \\
\hline & 3 & 1 & 0.00045 & & & 4 & 0.00037 & & & 7 & 1.16149 & & & 2 & 0.00002 \\
\hline & & 2 & 0.00196 & & & 5 & 0.00092 & 8 & 1 & 1 & 0.74842 & & & 3 & 0.00002 \\
\hline & & 3 & 1.00000 & & & 6 & 1.15689 & & 2 & 1 & 0.00057 & & & 4 & 0.00012 \\
\hline & 4 & 1 & 0.00012 & 7 & 1 & 1 & 0.75655 & & & 2 & 0.82607 & & & 5 & 0.00051 \\
\hline & & 2 & 0.00066 & & 2 & 1 & 0.00072 & & 3 & 1 & 0.00025 & & & 6 & 0.00056 \\
\hline & & 3 & 0.00196 & & & 2 & 0.84375 & & & 2 & 0.00117 & & & 7 & 0.00062 \\
\hline & & 4 & 1.08541 & & 3 & 1 & 0.00026 & & & 3 & 0.90056 & & & 8 & 1.16489 \\
\hline
\end{tabular}
bivariate distribution.

Table 1: Means of the concomitants of ORSS for Gumbel's bivariate exponential distribution, $\mu_{r: n}^{\text {ORSS }}(1 \leq r \leq n)$

Table 2: Variances and covariances of the concomitants of ORSS for Gumbel's bivariate exponential distribution, $\sigma_{r, s: n}^{\text {ORSS }}(1 \leq r$ 
We simulated the values of ${ }_{\text {ORSSS }} \mu_{[r, r: n]}$ and $\sigma_{[r, s: n]}^{\text {ORSS }}$ for $n=$ $1,2, \cdots, 8$ and $1 \leq r \leq s \leq n$ based on Monte Carlo simulations from the Gumbel's bivariate exponential distribution when $\alpha=0.5$ and $\beta_{2}=1$. The results are displayed in Table 1 and 2 for different choices of $n$.

\section{Conclusion}

In this study we investigated the distribution theory of concomitants of ORSS from the FGM bivariate distribution family. Also, we derived an analytical expression of the joint distribution of concomitants of two ORSS and find their single and product moments alongside the correlation coefficient. The family considered in this study is widely used in practice and gives more flexibility to model various types of data as well as it includes several important bivariate distributions. The Gumbel's bivariate exponential distribution is used as an example of the FGM bivariate family. Finally, we simulated the values of the means, variances and covariances of the concomitants of ORSS based on Monte Carlo simulations.

\section{Acknowledgement}

I hereby express my sincere thanks to the anonymous referees who read my earlier manuscript and gave valuable suggestions which led to a improvement in the paper.

\section{Ethics}

This article and all findings are original and contains unpublished material.

\section{References}

Adatia, A., 2000. Estimation of parameters of the halflogistic distribution using generalized ranked set sampling. Comput. Stat. Data Anal., 33: 1-13. DOI: 10.1016/S0167-9473(99)00035-3

Alodat, M.T. and O.A. Al-Sagheer, 2007. Estimation of location and scale parameters of using ranked set sampling. J. Applied Stat. Sci., 15: 245-252.

Al-Saleh, M.F. and A.I. Al-Omari, 2002. Multistage ranked set sampling. J. Stat. Plann. Inference, 102: 273-286. DOI: 10.1016/S0378-3758(01)00086-6

Al-Saleh, M.F. and H.M. Samawi, 2010. On estimating the odds using moving extreme ranked set sampling. Stat. Methodol., 7: 133-140. DOI: 10.1016/j.stamet.2009.11.004

Arnold, B.C., N. Balakrishnan and H.N. Nagaraja, 1992. A First Course in Order Statistics. 1st Edn., Wiley, New York, ISBN-10: 0471574163, pp: 304.

Bairamov, I.G. and M. Bekci, 1999. Concomitant of order statistics in FGM type bivariate uniform distributions. J. Turkish Stat. Assoc., 2: 135-144.
Balakrishnan, N., 2008. Permanents, order statistics, outliers and robustness. Revista Matematica Complutense, 20: 7-107.

Balakrishnan, N. and T. Li, 2008. Ordered ranked set samples and applications to inference. J. Stat. Plann. Inference, 138: 3512-3524. DOI: $10.1016 /$ j.jspi.2005.08.050

Balasubramanian, K. and M.I. Beg, 1997. Concomitant of order statistics in the bivariate exponential distributions of Marshall and Olkin. Calcutta Stat. Assoc. Bull., 46: 109-115.

Beg, M.I. and M. Ahsanullah, 2008. Concomitants of generalized order statistics from Farlie-GumbelMorgenstern distributions. Stat. Methodol., 5: 1-20. DOI: 10.1016/j.stamet.2007.04.001

Castillo, E., 1988. Extreme Value Theory in Engineering. 1st Edn., Academic Press, Boston, ISBN-10: 0121634752, pp: 389.

David, H.A. and H.N. Nagaraja, 1998. Concomitants of Order Statistics. In: Handbook of Statistics, Balakrishnan, N. and C.R. Rao (Eds.), pp: 487-513.

David, H.A. and H.N. Nagaraja, 2003. Order Statistics. 3rd Edn, Wiley, New York, ISBN-10: 0471389269, pp: 488.

Do, K.A. and P. Hall, 1991. Distribution theory using concomitants of order statistics with application to Monte Carlo simulation for the bootstrap. J. Royal Stat. Society B, 54: 595-607.

Domma, F. and S. Giordano, 2015. Concomitants of $m$ generalized order statistics from generalized FarlieGumbel-Morgenstern distribution family. J. Comput. Applied Math., 294: 413-435. DOI: $10.1016 /$ j.cam.2015.08.022

Farlie, D.J.G., 1960. The performance of some correlation coefficients for a general bivariate distribution. Biometrika, 47: 307-323. DOI: $10.1093 /$ biomet/47.3-4.307

Gross, A.L., 1973. Prediction in future samples studied in terms of the gain from selection. Psychometrika, 38: 151-171. DOI: 10.1007/BF02291111

Gumbel, E.J., 1960. Bivariate exponential distributions. J. Am. Stat. Assoc., 55: 698-707. DOI: $10.1080 / 01621459.1960 .10483368$

Halls, L.S. and T.R. Dell, 1966. Trial of ranked set sampling for gorage yields. Forest Sci., 12: 22-26.

Johnson, N.L. and S. Kott, 1975. On some generalized Farlie-Gumbel-Morgenstern distributions. Commun. Stat. Theory Meth., 4: 415-427. DOI: $10.1080 / 03610927508827258$

Johnson, N.L. and S. Kotz, 1977. On some generalized Farlie-Gumbel-Morgenstern distributions-II: Regression, correlation and further generalizations. Commun. Stat. Theory Meth., 6: 485-496. DOI: $10.1080 / 03610927708827509$ 
Kadilar, C., Y. Unyazici and H. Cingi, 2009. Ratio estimator for the population mean using ranked set sampling. Stat. Papers, 50: 301-309.

DOI: $10.1007 / \mathrm{s} 00362-007-0079-y$

Kotb, M.S., 2016. Bayesian prediction bounds for the exponential-type distribution based on ordered ranked Set rampling. Economic Quality Control, 31: 45-54.

Kotb, M.S. and M.Z. Raqab, 2017. Bayesian inference and prediction of the Rayleigh distribution based on ordered ranked set sampling. Commun. Stat. Simulat. Comput.

Martin, W.L., T. Shank, G. Oderwald and D.W. Smith, 1980. Evaluation of ranked set sampling for estimating shrub phytomass in application Oak forest. School of Forestry and Wildlife Recourses VPI and SU Blackburg, VA.

McIntyre, G.A., 1952. A method for unbiased selective sampling using ranked sets. Australian J. Agric. Res., 3: 385-390. DOI: 10.1071/AR9520385

Mohie El-Din, M.M., M.S. Kotb, E.F. Abd-Elfattah and H.A. Newer, 2017. Bayesian inference and prediction of the Pareto distribution based on ordered ranked set sampling. Commun. Stat. Theory Meth., 46: 6264-6279

DOI: $10.1080 / 03610926.2015 .1124118$

Mohie El-Din, M.M., M.S. Kotb and H.A. Newer, 2015. Bayesian estimation and prediction for Pareto distribution based on ranked set sampling. J. Stat. Applic. Probability, 4: 211-221.

Morgentern, D., 1956. Einfache beispiele zweidimensionaler verteilunngen. Mitteilungsblatt fur Mathemtische Statistik, 8: 234-235.

O'Connel, M.J. and H.A. David, 1976. Order statistics and their concomitants in some double sampling situations. Essays in Probability and Statistics, Shinko Tsusho.

Ozturk, O., 2011. Parametric estimation of location and scale parameters in ranked set sampling. J. Stat. Plann. Inference 141: 1616-1622.

DOI: $10.1016 /$ j.jspi.2010.11.019
Sadek, A., K.S. Sultan and N. Balakrishnan, 2015. Bayesian estimation based on ranked set sampling using asymmetric loss function. Bull. Malaysian Math. Sci. Society, 38: 707-718. DOI: $10.1007 / \mathrm{s} 40840-014-0045-5$

Salehi, M., J. Ahmadi and N. Balakrishnan, 2015. Prediction of order statistics and record values based on ordered ranked set sampling. J. Stat. Comput Simulat., 85: 77-88. DOI: $10.1080 / 00949655.2013 .803194$

Samawi, H.M., M.S. Ahmed and W. Abu-Dayyeh, 1996. Estimating the population mean using extreme ranked set sampling. Biometrical J., 38: 577-586. DOI: $10.1002 /$ bimj.4710380506

Shaibu, A.B. and H.A. Muttlak, 2004. Estimating the parameters of normal, exponential and gamma distributions using median and extreme ranked set samples. Statistica, 64: 75-98.

Sinha, B.K., K.S. Bikas and S. Purkayastha, 1996. On some aspects of ranked set sampling for estimation of normal and exponential parameters. Stat. Decisions, 14: 223-240.

DOI: $10.1524 /$ strm. 1996.14.3.223

Stokes, S.L., 1980. Estimation of variance using judgment ordered ranked set samples. Biometrics, 36: 35-42. DOI: 10.2307/2530493

Takahasi, K. and K. Wakimoto, 1968. On unbiased estimates of the population mean based on the sample stratified by means of ordering. Annals Inst. Stat. Math., 20: 1-31. DOI: 10.1007/BF02911622

Yang, S.S., 1977. General distribution theory of the concomitants of order statistics. Annals Stat., 5: 996-1002.

Yeo, W.B. and H.A. David, 1984. Selection through an associated characteristic with applications to the random effects model. J. Am. Stat. Assoc., 79: 399-405. DOI: $10.2307 / 2288282$

$\mathrm{Yu}$, P., 2001. Nonparametric estimation in ranked set sampling with concomitant variables. Proceedings of the Combinatorics and Related Areas and the Eighth International Conference of Forum for Interdisciplinary Mathematics, (FIM' 01). 